\title{
Impact of Fatigue on Outcomes in the Hemodialysis (HEMO) Study
}

\author{
Manisha Jhamb ${ }^{a}$ Francis Pike ${ }^{b}$ Sarah Ramer ${ }^{a}$ Christos Argyropoulos ${ }^{a}$ \\ Jennifer Stee ${ }^{c, d}$ Mary Amanda Dew ${ }^{b, c}$ Steven D. Weisbord ${ }^{\text {a, e }}$ Lisa Weissfeld ${ }^{b}$ \\ Mark Unruh ${ }^{\mathrm{a}}$ \\ ${ }^{a}$ Renal-Electrolyte Division, Departments of ${ }^{b}$ Biostatistics, and ${ }^{c}$ Psychiatry, University of Pittsburgh Medical Center, \\ dUniversity of Pittsburgh School of Medicine, Liver Cancer Center, Department of Surgery, Starzl Transplantation \\ Institute, and ${ }^{\mathrm{e}}$ Renal Section and Center for Health Equity Research and Promotion, VA Pittsburgh Healthcare, \\ Pittsburgh, Pa., USA
}

\section{Key Words}

End-stage renal disease $\cdot$ Fatigue $\cdot$ Vitality $\cdot$ Health-related quality of life

\begin{abstract}
Background: Fatigue is a common debilitating symptom in chronic kidney disease patients on maintenance hemodialysis. However, little is known about its pathogenesis and association with survival. Methods: This study examines the correlates and outcomes of fatigue among 1,798 hemodialysis patients enrolled in the HEMO study. Fatigue was as sessed using the SF-36 vitality scale. Multivariable analysis was used to assess independent associations of demographic and clinical characteristics with baseline fatigue and longitudinal changes in fatigue. The association of fatigue with all-cause and cause-specific mortality and cardiac hospitalizations was also assessed. Results: Higher index of coexistent diseases (ICED) score, diabetes, non-African-American race, lower serum albumin, use of medications for sleep and poor sleep quality were found to be significantly associated with more fatigue at baseline. In longitudinal analyses, patients who were older, had been on dialysis longer, had higher ICED score, and reported using medications for sleep were more likely to experience worsening fatigue, whereas higher
\end{abstract}

serum albumin was strongly associated with an improvement in level of fatigue. A 10-point increase in vitality score was associated with $10 \%$ increase in mean survival $(p<$ 0.0001). Conclusions: Demographic and clinical factors have significant associations with fatigue, which itself predicts mortality. Improving fatigue in the end-stage renal disease population may positively impact patient well-being and survival.

Copyright $\odot 2011$ S. Karger AG, Basel

\section{Introduction}

Fatigue is a common and often unrecognized symptom in patients with end-stage renal disease (ESRD) undergoing maintenance dialysis [1-3]. Various studies have reported that fatigue affects 60 to as many as $97 \%$ of dialysis patients $[1,4,5]$. Despite its wide prevalence in the ESRD population, renal providers are largely unaware of the presence and severity of this debilitating symptom [6]. Not only does fatigue severely impair physical and social functioning, it has been associated with lower quality of life and premature death in patients on chronic hemodialysis (HD) [7-9]. Fatigue's importance is further underscored by the results of a cross-sectional study in which

\section{KARGER}

Fax +4161306 1234 E-Mail karger@karger.ch www.karger.com

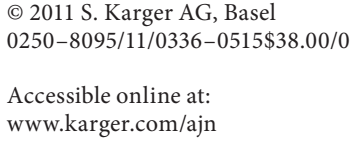

Mark Unruh, MD, MSc
University of Pittsburgh School of Medicine
200 Lothrop Street, PUH C-1111
Pittsburgh, PA 15213 (USA)
Tel. +1 412647 2571, E-Mail unruh@ @itt.edu 
94\% of the patients would accept more frequent dialysis if it would increase their energy level, but only 19\% would do so for an increase in survival up to 3 years [10].

However, despite advances in the knowledge and practice of delivering dialysis and improving biochemical markers of uremia, our ability to improve this critical patient-centered outcome remains very limited. The principal obstacles to improving our understanding and treatment of fatigue in ESRD patients are the relative paucity of data on its correlates and clinical implications, conflicting findings and a dearth of longitudinal data on the potentially modifiable correlates. Multiple physiologic, psychological, sociodemographic and treatment-related factors are likely associated with fatigue, but their complex and reciprocal interactions with fatigue are poorly understood $[1,2,11,12]$. If fatigue influences healthrelated quality of life (HRQOL) and/or survival, there would be a strong rationale for identification of fatigue and its correlates early in HD patients. Doing so may enable clinicians to treat modifiable risk factors early and thereby improve outcomes.

To this end, we examined the correlates of fatigue as well as its association with HRQOL, all-cause and causespecific mortality, and cardiac hospitalizations in prevalent HD patients in the HEMO Study. Although the effect of HD dose and flux on HRQOL in the HEMO study has been reported previously [13], the predictors of baseline fatigue and of longitudinal changes in fatigue in this wellcharacterized, multi-center, diverse cohort of maintenance HD patients have not been evaluated. Additionally, ours is the first study that examines longitudinal changes in fatigue and its association with survival.

\section{Methods}

\section{Study Participants}

The HEMO Study was a randomized controlled trial of 1,846 maintenance HD patients at 15 clinical centers comprising 72 dialysis units. It was designed to evaluate the effects of HD dose and membrane flux on morbidity and mortality in patients undergoing chronic HD. Patients were randomized by dose (standard vs. high-dose eKt/V) and membrane type (high- or low-flux membranes). Patient eligibility criteria have been described previously [14]. Enrollment lasted from March 1995 to October 2000. The mean follow-up was $2.84 \pm 1.84$ years. The Institutional Review Boards at the 15 institutions approved the study protocol and all participants provided written informed consent.

\section{Assessment of HRQOL and Fatigue}

At randomization and annually during follow-up, HEMO study patients responded to HRQOL surveys as previously described [13]. Patients were administered the Kidney Disease
Quality of Life-Long Form (KDQOL-LF), which contains the Medical Outcome Study 36-item short-form (SF-36) questionnaire. The SF-36 yields eight multi-item scales that measure physical functioning, bodily pain, mental health, general health, vitality, role emotional, role physical and social functioning. The SF36 vitality scale has good psychometric properties; internal consistency and reliability in the ESRD population [15]. We used the vitality scale of the SF-36 as a measure of the continuum of fatigue for this analysis. Scores range from 0 to 100, with higher scores reflecting more vitality and less fatigue.

In order to limit the number of comparisons, we only examined the associations of vitality scores with SF-36 Physical Functioning and Mental Health scores, as these were a priori thought to be related to fatigue. The range for all scales is from 0 to 100 , with higher scores indicating better health. The KDQOL instrument was also used to assess sleep quality. The sleep questions (10 items) assess subjective sleep initiation and maintenance, as well as daytime somnolence.

\section{Classification of Cause-Specific Mortality}

The determination of cause-specific mortality in the HEMO study has been previously described [13]. The cause-specific infectious, cardiac, and cardiovascular deaths were selected for this report since these are the most common etiologies of death among HD patients.

\section{Selection of Covariates}

Factors previously found to be associated with fatigue in the general population and among dialysis patients were examined [2-4, 8]: (1) demographic and clinical: age, gender, race, education, body mass index (BMI), blood pressure, smoking status; (2) dialysis-related factors: duration of dialysis; (3) laboratory tests: calcium (mg/dl), phosphorus (mg/dl), hematocrit (mg/dl), and serum albumin (g/dl); (4) comorbidity: index of coexistent diseases (ICED) score (calculated after excluding diabetes mellitus as a comorbidity) and diabetes mellitus; (5) treatment arms: since these data were collected in the setting of a randomized trial, the treatment arms were included as covariates; (6) psychological factors: SF-36 Mental Health score and sleep quality; (7) medication use: angiotensin-converting enzyme inhibitor (ACE-I), betablockers, alpha-1 inhibitor, erythropoietin, and self-reported use of medications for sleep (we did not have information on specific medications used for this purpose).

\section{Statistical Analysis}

Patients were grouped into four categories (quartiles) based on baseline vitality score so that the outcome could be analyzed as an ordinal variable to allow for easier clinical interpretation. We assumed, a priori, that any observed association of other variables with vitality would be linear across the quartiles. The trend tests supported this assumption post hoc. Baseline demographic, socioeconomic and laboratory factors are described as means and standard deviations (SDs) for continuous variables and as frequencies for categorical variables. Candidates for a multivariable ordinal logistic model were obtained via single covariate ordinal logistic regression models with an inclusion criterion of $\mathrm{p}<0.2$. Stepwise selection models were then used to obtain predictors of fatigue after adjusting for known confounders and forcing age, sex, race, BMI, medication use (ACE-I, beta-blockers, alpha-1 inhibitor, erythropoietin, sleep medication), and interview status 
(completed questionnaires via interview versus self) into the models.

To analyze the association of baseline fatigue with subsequent hospitalizations and all-cause and cause-specific mortality, unadjusted and adjusted hazard rates were obtained using proportional hazards regression. The hazard rates were adjusted by including those variables significant from the stepwise selection procedure described above along with age, sex, race, BMI, medication use, interview status, flux group, and dose group.

Longitudinal changes in vitality, and its effect on all-cause mortality were assessed via joint modeling. Joint models simultaneously assess the impact of changes in vitality on survival over time, while accounting for potentially informative missing vitality score data due to censoring and patient death. The model we used assigns a unique random intercept and slope to each patient which captures linear changes in vitality score over time. This 'true', but unobserved vitality is then used to adjust the survival experience which was assumed to follow a Weibull distribution. The degree of association between the 'true' vitality scores and survival can be interpreted as the change in mean survival per unit change in vitality score [16]. A linear trend test for the vitality quartiles was also computed, with vitality as a continuous variable in the model. The estimates of association obtained from the joint modeling of vitality and all-cause mortality are reported.

\section{Results}

\section{Baseline Patient Characteristics by Levels of Fatigue}

Baseline characteristics of patients with different levels of fatigue are shown in table 1 . The range of vitality scores among these prevalent HD patients was $0-100$ with the mean being $50.0 \pm 21.8 \mathrm{SD}$. A total of 1,308 (72.7\%) patients in our study had a baseline vitality score below the community-based average score of 60.9 \pm 20.9 [17]. Patients with a higher level of fatigue were more likely to be older, non-African-American, unemployed, and diabetic; they had more severe comorbid illness (higher ICED score) and slightly lower serum albumin levels; they had a higher likelihood of using medication for sleep and being administered the KDQOL-LF survey by an interviewer (as opposed to self-completion). Those with higher fatigue at baseline also reported a significantly lower HRQOL in the domains of mental health, physical functioning and sleep quality. There were no differences in serum creatinine, BMI, smoking status or duration of dialysis among the vitality quartiles. In addition, we found no significant differences in vitality scores across different strata of hematocrit (data not shown). Notably, there was a relatively narrow range of hematocrit values among patients in different quartiles.

Correlates of Fatigue and Outcomes in HD Patients

\section{Independent Predictors of Fatigue}

In multivariable analysis, African-American race was independently associated with less fatigue, as was higher serum albumin level. Higher ICED score, diabetes and use of sleep medications were independent predictors of more fatigue. Longer duration of dialysis also predicted less fatigue, although the effect size was small (table 2).

\section{Association of Fatigue with Survival}

Patients with baseline vitality scores in the first, second and third quartile were compared with those in the highest quartile (score $\geq 65$ ). The association of fatigue with all-cause mortality, cause-specific mortality, first cardiac hospitalization and the composite outcome of first cardiac hospitalization or cardiac death is shown in table 3. Patients in the lowest two vitality quartiles had a significantly higher risk of death from any cause as depicted in figure 1 (crude HR: 1.97, 95\% CI 1.63-2.36, and 1.33: 95\% CI 1.10-1.60). The risk of death was attenuated but remained significant for the lowest vitality quartile after adjusting for age, sex, race, BMI, ICED, duration of dialysis, albumin, treatment groups (flux and $\mathrm{Kt} / \mathrm{V}$ group), medication use and interviewed status (HR: 1.37, 95\% CI 1.12-1.67). There was a nonsignificant trend toward a higher hazard observed in the second and third quartile in the adjusted models.

We noted a trend towards increasing risk of death from cardiac death, infectious diseases and first cardiac hospitalization with worsening fatigue, but this was not statistically significant in the adjusted models. There was, however, a significantly increased risk for the composite outcome of first cardiac hospitalization or cardiac death with worsening fatigue (adjusted HR 1.27, 95\% CI 1.041.56; table 3).

\section{Longitudinal Changes in Fatigue and Its Association with All-Cause Mortality}

Results of this joint modeling are presented in table 4. Follow-up data for a median of 1.02 years were available for 1,763 patients. Patients who were older, had higher ICED score, reported using sleep medications and had been on dialysis for longer duration were more likely to experience worsening of fatigue over time. African-Americans and patients with higher serum albumin had improvement in fatigue over time (table 4). The adjusted estimate of the model association of longitudinal change in fatigue with survival was 0.01 ( $\mathrm{p}<0.0001$, 95\% CI $0.01-$ 0.02 ; table 4). Transforming this estimate as $100 \cdot \exp (\mathrm{es}-$ timate -1 ) implies a one-unit increase in vitality score is associated with a $1.00 \%$ increase in mean survival. 
Table 1. Patient characteristics by baseline level of fatigue

\begin{tabular}{|c|c|c|c|c|c|c|}
\hline \multirow[t]{2}{*}{ Characteristic } & \multirow{2}{*}{$\begin{array}{l}\text { Total } \\
(\mathrm{n}=1,798)\end{array}$} & \multicolumn{4}{|c|}{ Vitality quartile ${ }^{a}$} & \multirow[t]{2}{*}{$\mathrm{p}$ value $^{\mathrm{b}}$} \\
\hline & & $\begin{array}{l}1(\mathrm{VT} \text { score } \\
<35 \\
\mathrm{n}=466)\end{array}$ & $\begin{array}{l}2(\text { VT score } \\
\geq 35 \text { to }<50 \\
\mathrm{n}=531)\end{array}$ & $\begin{array}{l}3(\text { VT score } \\
\geq 50 \text { to }<65 \\
\mathrm{n}=310)\end{array}$ & $\begin{array}{l}4 \text { (VT score } \\
\geq 65 \\
\mathrm{n}=491)\end{array}$ & \\
\hline SF-36 vitality subscale & $50.0 \pm 21.8$ & $21.6 \pm 11.4$ & $46.2 \pm 4.2$ & $57.5 \pm 2.6$ & $76.3 \pm 9.5$ & $<0.001$ \\
\hline Age, years & $57.5 \pm 14.0$ & $59.4 \pm 13.9$ & $58.2 \pm 13.3$ & $56.5 \pm 14.8$ & $56.4 \pm 14.3$ & 0.0003 \\
\hline Male, \% & 43.7 & 43.1 & 44.6 & 38.4 & 46.4 & 0.57 \\
\hline African-American, \% & 63.7 & 52.2 & 62.7 & 69.4 & 71.5 & $<0.001$ \\
\hline Currently working, \% & 9.4 & 4.8 & 10.3 & 8.5 & 13.7 & $<0.001$ \\
\hline Less than 12 th-grade education, $\%$ & 37.7 & 37.4 & 38.7 & 37.2 & 37.3 & 0.87 \\
\hline Married, \% & 37.7 & 41.9 & 36.7 & 36.1 & 35.8 & 0.05 \\
\hline Currently smoking, \% & 17.5 & 16.6 & 19.6 & 15.2 & 17.1 & 0.83 \\
\hline $\begin{array}{l}\text { Dialysis characteristics } \\
\text { Time on dialysis, years } \\
\text { High Kt/V, \% } \\
\text { High flux, \% }\end{array}$ & $\begin{array}{l}3.9 \pm 4.4 \\
49.8 \\
49.9\end{array}$ & $\begin{array}{l}3.6 \pm 4.1 \\
50.2 \\
49.1\end{array}$ & $\begin{array}{l}3.8 \pm 4.2 \\
51.6 \\
49.7\end{array}$ & $\begin{array}{l}4.3 \pm 4.7 \\
48.1 \\
49.0\end{array}$ & $\begin{array}{l}4.1 \pm 4.6 \\
48.9 \\
51.3\end{array}$ & $\begin{array}{l}0.05 \\
0.51 \\
0.55\end{array}$ \\
\hline $\begin{array}{l}\text { Health and comorbidity characteristi } \\
\text { Systolic blood pressure, } \mathrm{mm} \mathrm{Hg} \\
\text { Diastolic blood pressure, } \mathrm{mm} \mathrm{Hg} \\
\text { BMI } \\
\text { Diabetic, \% } \\
\text { ICED score }{ }^{\text {c }} \% \\
\quad 0 \text { or } 1 \\
\quad 2 \\
\quad 3\end{array}$ & $\begin{array}{l}151.1 \pm 25.5 \\
81.3 \pm 15.3 \\
25.5 \pm 5.3 \\
44.8\end{array}$ & $\begin{array}{l}151.7 \pm 27.4 \\
80.5 \pm 15.7 \\
25.5 \pm 5.3 \\
53.4\end{array}$ & $\begin{array}{l}150.5 \pm 26.7 \\
80.7 \pm 15.2 \\
25.7 \pm 5.6 \\
44.1 \\
37.9 \\
31.6 \\
30.5\end{array}$ & $\begin{array}{l}150.7 \pm 24.8 \\
81.5 \pm 14.8 \\
25.2 \pm 5.0 \\
39.4\end{array}$ & $\begin{array}{l}151.1 \pm 22.9 \\
82.3 \pm 15.4 \\
25.5 \pm 5.2 \\
40.9 \\
44.6 \\
28.9 \\
26.5\end{array}$ & $\begin{array}{c}0.81 \\
0.05 \\
0.83 \\
<0.0001 \\
<0.0001\end{array}$ \\
\hline $\begin{array}{l}\text { Laboratory values } \\
\text { Albumin, mg/dl } \\
\text { Creatinine, mg/dl } \\
\text { Hematocrit, \% } \\
\text { iPTH, pg/ml } \\
\text { Phosphorus, mg/dl }\end{array}$ & $\begin{array}{r}3.6 \pm 0.4 \\
10.3 \pm 3.0 \\
33.6 \pm 4.6 \\
5.1 \pm 1.2 \\
5.8 \pm 1.9\end{array}$ & $\begin{array}{r}3.5 \pm 0.3 \\
10.3 \pm 3.1 \\
34.0 \pm 4.5 \\
5.1 \pm 1.2 \\
5.8 \pm 1.8\end{array}$ & $\begin{array}{r}3.6 \pm 0.4 \\
10.3 \pm 2.8 \\
33.4 \pm 4.4 \\
5.2 \pm 1.2 \\
5.8 \pm 1.9\end{array}$ & $\begin{array}{r}3.7 \pm 0.4 \\
10.3 \pm 3.0 \\
33.4 \pm 4.9 \\
5.2 \pm 1.2 \\
5.8 \pm 2.1 \\
\end{array}$ & $\begin{array}{r}3.7 \pm 0.3 \\
10.3 \pm 3.2 \\
33.5 \pm 4.7 \\
5.1 \pm 1.2 \\
5.8 \pm 1.9\end{array}$ & $\begin{array}{c}<0.001 \\
0.92 \\
0.11 \\
0.74 \\
0.98\end{array}$ \\
\hline $\begin{array}{l}\text { Medications used, \% } \\
\text { ACE inhibitor } \\
\text { Beta-blocker } \\
\text { Alpha-1 blocker } \\
\text { EPO } \\
\text { Sleeping pill }\end{array}$ & $\begin{array}{r}25.1 \\
30.0 \\
6.7 \\
90.7 \\
25.1\end{array}$ & $\begin{array}{r}26.5 \\
28.5 \\
7.6 \\
91.3 \\
36.3\end{array}$ & $\begin{array}{r}22.5 \\
27.8 \\
5.5 \\
89.9 \\
26.4\end{array}$ & $\begin{array}{r}24.4 \\
34.5 \\
6.5 \\
89.9 \\
23.9\end{array}$ & $\begin{array}{r}26.3 \\
31.1 \\
6.9 \\
91.4 \\
14.1\end{array}$ & $\begin{array}{c}0.88 \\
0.15 \\
0.81 \\
0.99 \\
<0.001\end{array}$ \\
\hline $\begin{array}{l}\text { Psychosocial characteristics } \\
\text { SF-36 Physical Functioning } \\
\text { SF-36 Mental Health } \\
\text { KDQOL sleep quality score } \\
\text { Interviewed, \% }\end{array}$ & $\begin{array}{l}48.1 \pm 26.9 \\
71.6 \pm 19.3 \\
58.8 \pm 22.7 \\
40.9\end{array}$ & $\begin{array}{l}33.0 \pm 23.3 \\
59.8 \pm 20.8 \\
47.7 \pm 22.3 \\
39.6\end{array}$ & $\begin{array}{l}44.7 \pm 24.5 \\
67.9 \pm 16.4 \\
56.2 \pm 21.1 \\
48.7\end{array}$ & $\begin{array}{l}54.3 \pm 23.7 \\
76.1 \pm 15.3 \\
61.4 \pm 21.3 \\
36.2\end{array}$ & $\begin{array}{l}62.5 \pm 26.1 \\
84.0 \pm 14.3 \\
70.5 \pm 19.3 \\
30.9\end{array}$ & $\begin{array}{c}<0.0001 \\
<0.0001 \\
<0.0001 \\
0.008\end{array}$ \\
\hline
\end{tabular}

iPTH = Intact parathyroid hormone; ACE = angiotensin-converting enzyme; EPO = erythropoietin. Values are means \pm standard deviations. ${ }^{a}$ Higher vitality score reflects less fatigue. ${ }^{b}$ Jonckheere-Terpstra test of trend for categorical variables and generalized linear models with linear trend for continuous variables. ${ }^{c}$ ICED score calculated after excluding diabetes mellitus as a comorbidity. 
Table 2. Predictors of fatigue at baseline via univariable and multivariable ordinal logistic regression

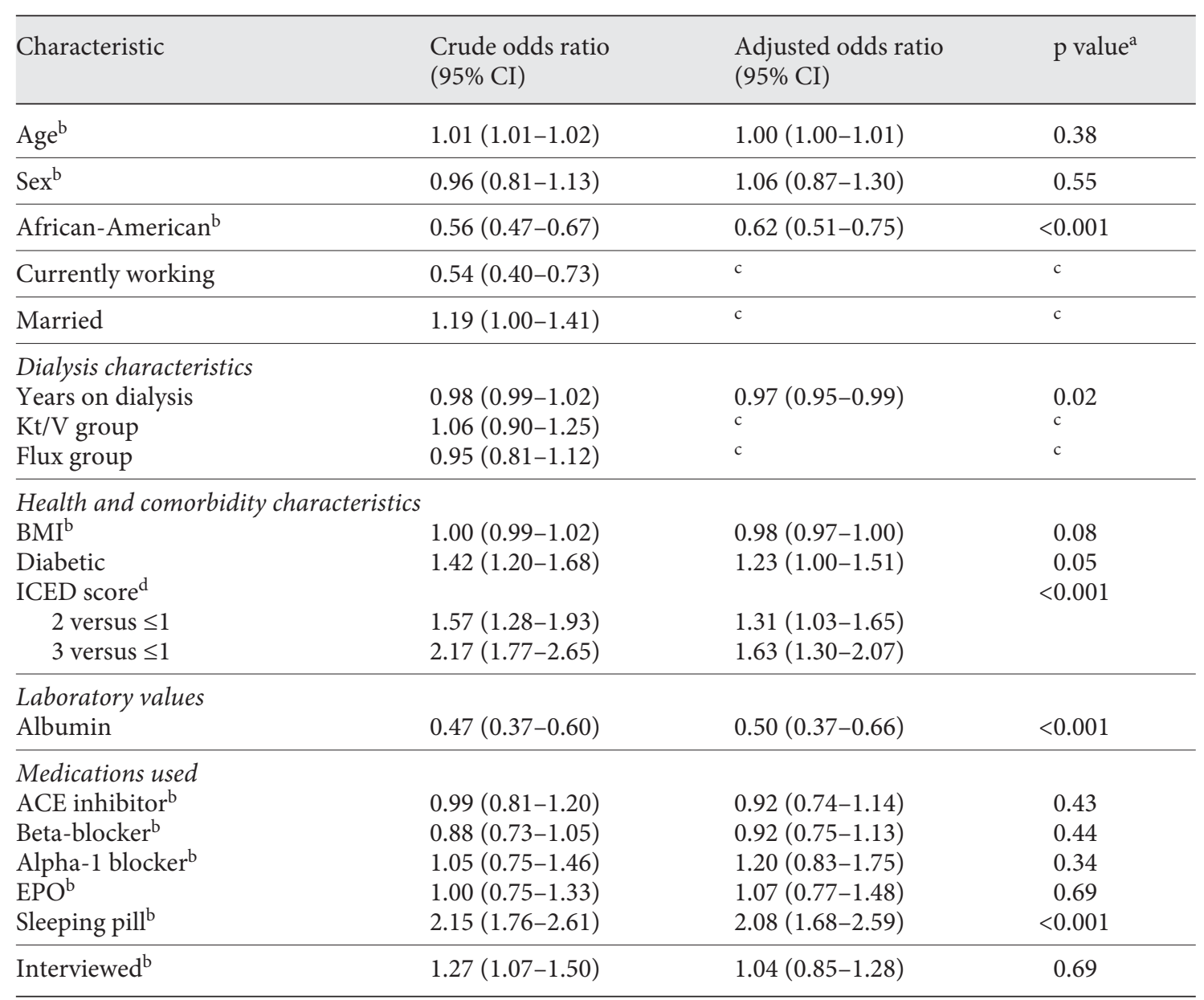

${ }^{a} \mathrm{p}$ values are for the adjusted model. ${ }^{\mathrm{b}}$ Forced into models. ${ }^{\mathrm{c}}$ Variable not significant at $\mathrm{p}<0.2$ in a stepwise selection procedure. ${ }^{\mathrm{d}}$ ICED score calculated after excluding diabetes mellitus as a comorbidity.

\section{Discussion}

Our findings demonstrate that maintenance HD patients commonly experience severe fatigue. The average vitality scores of these patients are lower than nearly every other chronic health condition [17]. More than 50\% of the patients in our study had a vitality score of less than 50 , which is comparable to those with severe depression [18], cancer patients undergoing chemotherapy [19], and those with lupus [20].

Our results identify some of the important correlates of fatigue, which can facilitate our understanding of the complex and interdependent relations of these with fatigue and overall HRQOL. Some of these may be potentially modifiable and may have important clinical impli- cations in developing interventions to mitigate fatigue in these patients. We observed that higher ICED score, presence of diabetes and use of sleep medications were associated with more fatigue at baseline. In contrast, increased number of years on dialysis, African-American race and having higher albumin were associated with less fatigue.

The importance of fatigue as an indicator of health in the HD population was further highlighted by the strong association of fatigue with HRQOL and survival in our study. We found that more severe fatigue at baseline was independently associated with an increased risk of allcause mortality, cardiac mortality and a composite of cardiac mortality or first cardiac hospitalization in maintenance HD patients, even after adjusting for traditional 
Table 3. Association of baseline fatigue with patient survival

\begin{tabular}{|c|c|c|c|c|}
\hline & $\begin{array}{l}\text { Crude HR } \\
(95 \% \mathrm{CI})^{\mathrm{a}}\end{array}$ & $\begin{array}{l}\text { Trend } \\
\text { test }^{c}\end{array}$ & $\begin{array}{l}\text { Adjusted HR } \\
(95 \% \mathrm{CI})^{\mathrm{b}}\end{array}$ & $\begin{array}{l}\text { Trend } \\
\text { test }^{c}\end{array}$ \\
\hline All-cause mortality & & $<0.0001$ & & 0.001 \\
\hline 3 rd quartile versus 4 th quartile & $1.11(0.89-1.39)$ & & $1.07(0.84-1.35)$ & \\
\hline 2nd quartile versus 4 th quartile & $1.33(1.10-1.60)$ & & $1.19(0.98-1.45)$ & \\
\hline 1st quartile versus 4 th quartile & $1.97(1.63-2.36)$ & & $1.37(1.12-1.67)$ & \\
\hline Cardiac death & & $<0.0001$ & & 0.04 \\
\hline 3 rd quartile versus 4 th quartile & $0.95(0.66-1.40)$ & & $0.97(0.65-1.46)$ & \\
\hline 2nd quartile versus 4 th quartile & $1.33(0.98-1.82)$ & & $1.14(0.82-1.59)$ & \\
\hline 1st quartile versus 4 th quartile & $2.01(1.49-2.73)$ & & $1.39(0.99-1.93)$ & \\
\hline Death from infectious disease & & 0.0004 & & 0.24 \\
\hline 3 rd quartile versus 4 th quartile & $0.96(0.58-1.59)$ & & $0.81(0.48-1.37)$ & \\
\hline 2nd quartile versus 4 th quartile & $1.40(0.94-2.08)$ & & $1.15(0.76-1.73)$ & \\
\hline 1st quartile versus 4 th quartile & $1.97(1.32-2.94)$ & & $1.21(0.79-1.86)$ & \\
\hline First cardiac hospitalization & & $<0.0001$ & & 0.10 \\
\hline 3 rd quartile versus 4 th quartile & $0.98(0.77-1.24)$ & & $0.93(0.73-1.19)$ & \\
\hline 2nd quartile versus 4 th quartile & $1.21(0.99-1.47)$ & & $1.08(0.88-1.33)$ & \\
\hline 1st quartile versus 4 th quartile & $1.60(1.31-1.94)$ & & $1.17(0.94-1.45)$ & \\
\hline First cardiac hospitalization or cardiac death & & $<0.0001$ & & 0.01 \\
\hline 3 rd quartile versus 4 th quartile & $0.97(0.78-1.22)$ & & $0.93(0.78-1.19)$ & \\
\hline 2nd quartile versus 4 th quartile & $1.22(1.01-1.47)$ & & $1.09(0.90-1.33)$ & \\
\hline 1st quartile versus 4 th quartile & $1.72(1.44-2.07)$ & & $1.27(1.04-1.56)$ & \\
\hline
\end{tabular}

${ }^{a}$ Univariate: no adjustment. ${ }^{b}$ Adjusted for baseline and model-selected covariates: vitality rank, ICED, age, sex, race, BMI, duration of dialysis, serum albumin, flux group, Kt/V group, medication use (ACE-I, betablockers, alpha-1 inhibitors, EPO, sleeping medication), interviewed. ${ }^{\mathrm{c}}$ Ordinal test of trend.

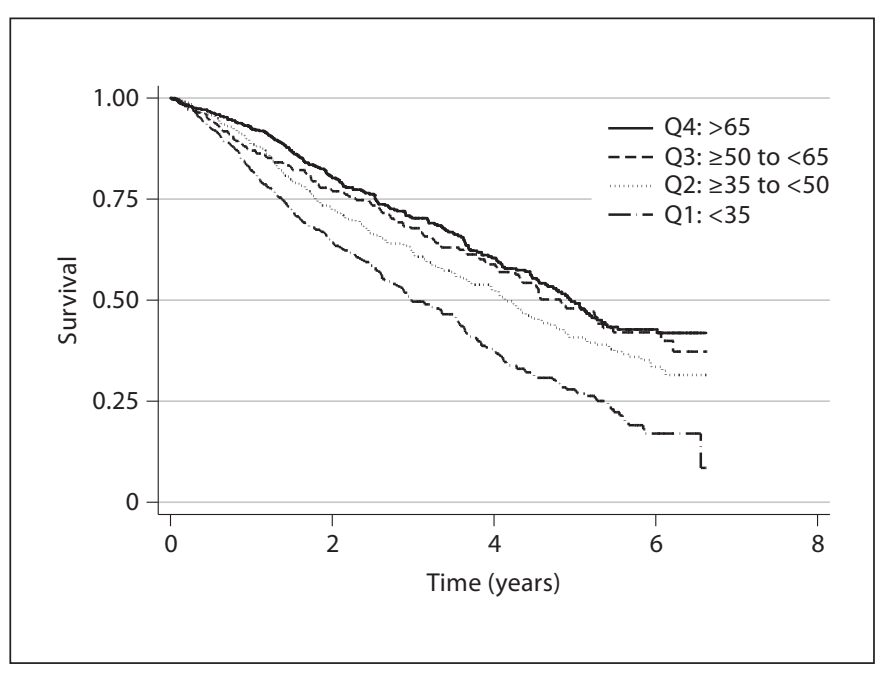

Fig. 1. All-cause mortality by vitality quartile. risk factors. These results build upon findings from prior studies in the general population [21] and HD patients [8, 9] and provide results that are more generalizable to maintenance HD patients in the USA as represented by the diverse, multicenter HEMO cohort.

Our results further extend these findings and demonstrate, for the first time, that not only baseline fatigue, but longitudinal changes in fatigue over time are also independently associated with mortality in HD patients. We found that every 10-point increase in vitality score over time increased the chances of survival by $10 \%$. The biological mechanisms through which fatigue is associated with survival are not well understood. Similar to the general population, metabolic, hemodynamic and immune responses such as abnormal lipid metabolism, decreased fibrinolysis, low heart-rate variability and higher levels of proinflammatory cytokines associated with fatigue may also contribute to increased risk of cardiovascular events and death in the ESRD population $[8,21]$. Thus, periodic assessment of fatigue, specially worsening of fatigue over 
Table 4. Joint model analysis of longitudinal change in fatigue and survival

\begin{tabular}{lcc}
\hline Characteristic & Joint model & \\
\cline { 2 - 3 } & longitudinal sub-model & survival sub-model \\
\hline Age, years & $-0.09(-0.16,-0.02)$ & $-0.02(-0.03,-0.02)$ \\
Sex & $0.43(-1.37,2.22)$ & $-0.10(-0.21,0.02)$ \\
Interview & $-1.04(-2.91,0.82)$ & $-0.20(-0.32,-0.09)$ \\
African-American & $6.85(5.03,8.68)$ & $0.27(0.16,0.38)$ \\
ICED (2) & $-3.61(-5.77,-1.45)$ & $-0.31(-0.46,-0.17)$ \\
ICED (3) & $-5.45(-7.62,-3.28)$ & $-0.46(-0.60,-0.31)$ \\
Duration, years & $0.07(-0.13,0.26)$ & $-0.01(-0.03,-0.00)$ \\
Serum albumin, mg/dl & $7.63(5.00,10.27)$ & $0.79(0.62,0.96)$ \\
BMI & $-0.01(-0.18,0.15)$ & $0.03(0.02,0.04)$ \\
Sleeping pill & $-7.61(-9.60,-5.62)$ & $-0.11(-0.23,0.01)$ \\
Time, years & $-1.57(-1.94,-1.21)$ & - \\
Flux group & $0.79(-0.92,2.49)$ & $0.02(-0.08,0.13)$ \\
Kt/V group & $0.34(-1.37,2.05)$ & $0.06(-0.04,0.17)$ \\
Model association & & $0.01(0.01,0.02)$ \\
\hline
\end{tabular}

a Transforming the adjusted estimate of the model association of longitudinal change in fatigue with survival as $100 \cdot \exp ($ estimate -1$)$ implies a one-unit increase in vitality score is associated with a $1.00 \%$ increase in mean survival.

time, may help identify patients who are at high risk of such adverse outcomes.

Our study demonstrated that higher albumin was strongly and independently associated with improved fatigue and survival over time. An increase in albumin by $1 \mathrm{~g} / \mathrm{dl}$ was associated with an increase in vitality score by 7.7 points and $21 \%$ decrease in risk of death. Hypoalbuminemia, which is a marker of malnutrition-inflammation complex syndrome and a strong predictor of poor quality of life and cardiovascular death [22-25], may also similarly affect fatigue in maintenance HD patients. These findings are consistent with reports demonstrating that dialysis patients with laboratory evidence of malnutrition and/or inflammation have low levels of physical activity, higher fatigue and mortality $[1,2,8,26]$. Perhaps improving nutrition or increasing albumin levels by pharmacological interventions [27] in these patients will improve not only quantity but also quality of life by reducing fatigue.

In our study, the African-American HD patients had an improvement in fatigue on longitudinal follow-up as compared to non-African-American patients. These results are in concordance with findings from the Dialysis Morbidity and Mortality Study Wave 2, CHOICE and HEMO study, which demonstrated that African-Americans perceive lesser burden of disease than non-AfricanAmericans $[8,28,29]$. These findings suggest that race may play an important role in determining adaptation to dialysis.

We observed that patients with higher fatigue had significantly worse physical and mental HRQOL and poor sleep quality. Self-reported use of sleep medications was associated with higher baseline fatigue and predicted worsening of fatigue over time. It may be that people who were using sleep medications had poorer sleep quality and thus reported more fatigue, or that fatigue may be a side effect of these medications. Studies are needed to assess whether improving sleep quality (e.g. by using CPAP for patients with obstructive sleep apnea) will reduce fatigue in these patients.

One of the surprising and counterintuitive findings in our study was that longer dialysis vintage was significantly associated with lower fatigue at baseline. The patients in the HEMO study had been on dialysis for an average of 4 years and may represent a self-selected cohort of patients who are healthier than those who died before being enrolled in the study. This association was not seen on longitudinal follow-up which is less susceptible to survivorship bias.

Our study has a number of strengths from using participants in the HEMO study who reflect a diverse, multicenter sample of maintenance HD patients. The HEMO study facilitated highly successful longitudinal data collection, making the results of our longitudinal analysis 
very important and reliable. Also, ours is the largest study assessing fatigue and survival in this patient population. Additionally, the HEMO study collected data on causespecific mortality and cardiac hospitalizations, thus allowing us to analyze the effect of fatigue on these specific outcomes. By using the novel approach of joint modeling, we accounted for the association between the longitudinal change in fatigue and survival when estimating the effect of fatigue and survival. This modeling approach also addresses selection and survivor bias as mentioned above.

There are several limitations to our study. First, the HEMO study enrolled only prevalent dialysis patients, and thus the findings may not generalize to patients who are initiating dialysis. However, our findings do provide a glimpse into how patients can expect to feel once they are established on maintenance dialysis. Moreover, we found no differences in baseline fatigue when stratified by dialysis vintage. Additionally, although the HEMO study enrolled participants from 1995 to 2000, our results are still likely valid today as few, if any, advancements in the technique of delivering HD have shown to improve HRQOL in these patients [30]. Also, these are selected clinical trial patients, which may limit the generalizabil- ity of our findings. Second, depression was not assessed in the HEMO study. Depression, as well as the medications used to treat it, can affect fatigue and sleep and can be a potential confounding factor. Future studies should aim at measuring depression, and its treatment and fatigue concurrently. Third, the SF-36 vitality scale may fail to fully capture people with more severe fatigue. However, this instrument has been extensively used to assess fatigue in the general population and other chronic health conditions, allowing for comparison with these populations. Future work should consider instruments which reliably measure more severe fatigue.

In conclusion, the results of our study provide clinically relevant information on predictors of fatigue in the ESRD population. Some of these, such as sleep quality and albumin levels may be modifiable and amenable to interventions. Baseline fatigue and longitudinal changes in fatigue over time may influence the risk of death. Future work should focus on investigating the impact of potential pharmacological or exercise-based interventions to mitigate fatigue, such as those used for cancer-related fatigue [31,32]. This may help improve survival and HRQOL in this growing and chronically ill patient population.

\section{References}

1 Jhamb M, Weisbord SD, Steel JL, Unruh M: Fatigue in patients receiving maintenance dialysis: a review of definitions, measures, and contributing factors. Am J Kidney Dis 2008;52:353-365.

2 Bossola M, Luciani G, Tazza L: Fatigue and its correlates in chronic hemodialysis patients. Blood Purif 2009;28:245-252.

-3 Liu HE: Fatigue and associated factors in hemodialysis patients in Taiwan. Res Nurs Health 2006;29:40-50.

-4 Parfrey PS, Vavasour HM, Henry S, Bullock M, Gault MH: Clinical features and severity of nonspecific symptoms in dialysis patients. Nephron 1988;50:121-128.

5 Yngman-Uhlin P, Friedrichsen M, Gustavsson M, Fernstrom A, Edell-Gustafsson U: Circling around in tiredness: perspectives of patients on peritoneal dialysis. Nephrol Nurs J 2010;37:407-413.

-6 Weisbord SD, Fried LF, Mor MK, et al: Renal provider recognition of symptoms in patients on maintenance hemodialysis. Clin J Am Soc Nephrol 2007;2:960-967.

7 Bonner A, Wellard S, Caltabiano M: The impact of fatigue on daily activity in people with chronic kidney disease. J Clin Nurs 2010;19:3006-3015.
8 Jhamb M, Argyropoulos C, Steel JL, et al: Correlates and outcomes of fatigue among incident dialysis patients. Clin J Am Soc Nephrol 2009;4:1779-1786.

-9 Koyama H, Fukuda S, Shoji T, et al: Fatigue is a predictor for cardiovascular outcomes in patients undergoing hemodialysis. Clin J Am Soc Nephrol 2010;5:659-666.

10 Ramkumar N, Beddhu S, Eggers P, Pappas LM, Cheung AK: Patient preferences for incenter intense hemodialysis. Hemodial Int 2005;9:281-295.

-11 Kamimura MA, Draibe SA, Dalboni MA, et al: Serum and cellular interleukin-6 in haemodialysis patients: relationship with energy expenditure. Nephrol Dial Transplant 2007;22:839-844.

12 Bossola M, Luciani G, Giungi S, Tazza L: Anorexia, fatigue, and plasma interleukin-6 levels in chronic hemodialysis patients. Ren Fail 2010;32:1049-1054.

13 Unruh M, Benz R, Greene T, et al: Effects of hemodialysis dose and membrane flux on health-related quality of life in the HEMO Study. Kidney Int 2004;66:355-366.

14 Greene T, Beck GJ, Gassman JJ, et al: Design and statistical issues of the hemodialysis (HEMO) study. Control Clin Trials 2000;21: 502-525.
15 Meyer KB, Espindle DM, DeGiacomo JM, Jenuleson CS, Kurtin PS, Davies AR: Monitoring dialysis patients' health status. Am J Kidney Dis 1994;24:267-279.

16 Guo X, Carlin BP: Separate and joint modeling of longitudinal and event time data using standard computer packages. Am Stat 2004; 58:16-24.

17 Ware J, Snow K, Kosinski M, Gandek B: SF36 Health Survey Manual and Interpretation Guide. Boston, The Health Institute, New England Medical Center, 1993.

18 Yatham LN, Lecrubier Y, Fieve RR, Davis KH, Harris SD, Krishnan AA: Quality of life in patients with bipolar I depression: data from 920 patients. Bipolar Disord 2004;6: 379-385.

19 Adamsen L, Quist M, Andersen C, et al: Effect of a multimodal high intensity exercise intervention in cancer patients undergoing chemotherapy: randomised controlled trial. BMJ 2009;339:b3410.

20 Jolly M: How does quality of life of patients with systemic lupus erythematosus compare with that of other common chronic illnesses? J Rheumatol 2005;32:1706-1708. 
-21 Williams JE, Mosley TH Jr, Kop WJ, Couper DJ, Welch VL, Rosamond WD: Vital exhaustion as a risk factor for adverse cardiac events (from the Atherosclerosis Risk In Communities [ARIC] study). Am J Cardiol 2010;105: 1661-1665.

-22 Herselman M, Esau N, Kruger JM, Labadarios D, Moosa MR: Relationship between serum protein and mortality in adults on longterm hemodialysis: exhaustive review and meta-analysis. Nutrition 2010;26:10-32.

-23 Rambod M, Bross R, Zitterkoph J, et al: Association of malnutrition-inflammation score with quality of life and mortality in hemodialysis patients: a 5-year prospective cohort study. Am J Kidney Dis 2009;53:298309.

24 Kalantar-Zadeh K, Kopple JD, Block G, Humphreys MH: A malnutrition-inflammation score is correlated with morbidity and mortality in maintenance hemodialysis patients. Am J Kidney Dis 2001;38:12511263.
25 Kalantar-Zadeh K, Kopple JD, Humphreys $\mathrm{MH}$, Block G: Comparing outcome predictability of markers of malnutrition-inflammation complex syndrome in haemodialysis patients. Nephrol Dial Transplant 2004; 19: 1507-1519.

26 Anand S, Chertow GM, Johansen KL, et al: Association of self-reported physical activity with laboratory markers of nutrition and inflammation: the Comprehensive Dialysis Study. J Ren Nutr 2011, Epub ahead of print.

27 Kopple JD, Cheung AK, Christiansen JS, et al: OPPORTUNITY: a randomized clinical trial of growth hormone on outcome in hemodialysis patients. Clin J Am Soc Nephrol 2008;3:1741-1751.

28 Kutner NG, Zhang R, Brogan D: Race, gender, and incident dialysis patients' reported health status and quality of life. J Am Soc Nephrol 2005;16:1440-1448.
29 Unruh M, Miskulin D, Yan G, et al: Racial differences in health-related quality of life among hemodialysis patients. Kidney Int 2004;65:1482-1491.

30 Gabbay E, Meyer KB, Griffith JL, Richardson MM, Miskulin DC: Temporal trends in health-related quality of life among hemodialysis patients in the United States. Clin J Am Soc Nephrol 2010;5:261-267.

-31 Brown JC, Huedo-Medina TB, Pescatello LS, Pescatello SM, Ferrer RA, Johnson BT: Efficacy of exercise interventions in modulating cancer-related fatigue among adult cancer survivors: a meta-analysis. Cancer Epidemiol Biomarkers Prev 2011;20:123-133.

32 Peuckmann V, Elsner F, Krumm N, Trottenberg P, Radbruch L: Pharmacological treatments for fatigue associated with palliative care. Cochrane Database Syst Rev 2010; CD006788. 